\title{
"Warum sagt einem das niemand?" Pluralität und Reflexivität für die schulische (sozio-)ökonomische Bildung
}

\section{Marcel Beyer}

\section{Zusammenfassung}

Analog zur Bezugswissenschaft Ökonomik stellt sich auch in der universitären (sozio-)ökonomischen Lehrerbildung die Frage einer gelingenden Umsetzung und Etablierung von pluraler Ökonomik und epistemologischer Reflexion. Denn wenn angehende Lehrerinnen und Lehrer im Studium eine maßgeblich einseitige ökonomische Bildung erfahren, ihnen Alternativen im Denken und Handeln über Ökonomie vorenthalten werden, wie können diese dann Schülerinnen und Schüler befähigen, zentrale Probleme (Klimakrise, Ungleichheiten) multiperspektivisch zu befragen und anzugehen? Dieses Problem wird in meinem Beitrag einerseits vertieft, andererseits werden anhand eines fachdidaktischen Seminars in einem Bachelor-Studiengang konkrete Lösungsansätze für die Hochschullehre aufgezeigt. Dieses Seminar verfolgte das Ziel, Pluralität sowie Reflexivität zu den obersten Prämissen der fachwissenschaftlichen sowie fachdidaktischen Auseinandersetzung zu machen.

\section{Schlüsselbegriffe}

Sozioökonomische Bildung · Pluralität · Reflexivität · Paradigmenvielfalt · Ökonomisierung $\cdot$ Lehrerbildung $\cdot$ Neues ökonomisches Denken

\footnotetext{
M. Beyer $(\bowtie)$

Universität Bielefeld, Bielefeld, Deutschland

E-Mail: marcel.beyer@uni-bielefeld.de
} 


\section{$1 \quad$ Problemstellung}

„Was heißt Wirtschaften?““ (Riedel 2015, S. 204) wird in einem Schulbuch der Klassenstufe 7 im Fach Politik und Wirtschaft (Hessen) als Leitfrage gestellt. Richtig! Die eindeutige Antwort ist: „Wirtschaften heißt entscheiden“ (ebd., S. 206), doch ,gibt es eine Regel dafür, wie wir uns entscheiden? Im wirtschaftlichen Denken besteht die Regel darin, den Nutzen einer Handlung mit den Kosten, welche die Handlung verursacht, zu vergleichen." (ebd., eigene Hervorhebungen). Was sich in diesen einleitenden Definitionen exemplarisch zeigt, ist das einseitige Durchschlagen des Wirtschaftsverständnisses der Neoklassik. Andere Paradigmen etwa aus dem Fundus der Pluralen Ökonomik, die in der Wissenschaft kontrovers diskutiert werden, finden sich nicht. Zudem kann im genannten Beispiel Wirtschaften lediglich als Methode auf die Welt zu blicken verstanden werden, und ist nicht nach ihrem Gegenstandsbereich gefasst, in dem es beispielsweise um Produktions-, Reproduktions- und Verteilungsfragen (Hedtke 2008, S. 14) gehen würde. Kontroversität und Reflexivität werden damit deutlich unterlaufen.

Was diese skizzierte Problemlage für die universitäre Lehrerausbildung bedeutet und wie dieser im Rahmen des hier vorgestellten Seminars begegnet wurde, ist Kern des vorliegenden Beitrags.

\subsection{Das ,Was': einseitige ökonomische Bildung}

Als Problemlage identifiziert ist eine vielfach in Lehrplänen, Curricula und Lehrmitteln vorzufindende Einseitigkeit der ökonomischen Bildung, ausgerichtet auf die Etablierung einer einheitlichen wirtschaftswissenschaftlichen Perspektive (so die Kritik z. B. von Engartner et al. 2019). Diese Form der ökonomischen Bildung stellt sich als überwiegend abbilddidaktisch konzipiert und monoparadigmatisch organisiert dar: Inhalte und Zugriffe aus den Wirtschaftswissenschaften werden für die schulische Bildung reduziert übertragen (abbilddidaktisch) und fast ausschließlich unter dem Paradigma der Neoklassik (monoparadigmatisch) verhandelt. Nicht gesellschaftliche Probleme und Herausforderungen, sondern Inhalte der Bezugswissenschaft strukturieren damit den Unterricht und verengen die Auseinandersetzung der Schülerinnen und Schüler mit dem Gegenstandsbereich Wirtschaft. Dabei gibt es eine große Bandbreite an alternativen Ansätzen für die ökonomische Bildung: Plurale Ökonomik und Interdisziplinarität bieten vielfältige Möglichkeiten Methodenvielfalt und eine 
am Gegenstandsbereich Wirtschaft und der Lebenswelt von Schülerinnen und Schülern orientierte Bildung zu ermöglichen. Genau dies fehlt einer rein auf neoklassische Ökonomik reduzierten ökonomischen Bildung: die Auseinandersetzung mit Phänomenen der realen Welt (Pühringer und Bäuerle 2019).

Diese in heutigen Zeiten dringend notwendige Perspektivenpluralität, die einen Umgang mit den Herausforderungen unserer Zeit - hier seien nur exemplarisch Verteilungsfragen, globale Ungerechtigkeiten oder auch die Klimakrise genannt - ermöglicht, ist jedoch vielfach nicht für die schulische Bildung zum Thema Wirtschaft vorgesehen. Vielmehr steht hier die immer wieder angebrachte Forderung im Vordergrund: „Nicht das Fach ,Wirtschaft", sondern die Perspektive der Wirtschafts-Wissenschaft muss in deutschen Schulen breitenwirksam eingeführt werden" (Pies 2017, S. 2, Hervorhebung im Original). Schulpolitisch zeigt sich dies an Veränderungen von Bildungsplänen in den letzten Jahren, die vielfach mit einer, ,Verengung und Vervolkswirtschaftlichung der ökonomischen Bildung" (Hedtke 2012, S. 18) einhergehen. Betroffen sind sowohl Monofachkonstruktionen ${ }^{1}$ eines Faches Wirtschaft als auch Inhalts- und Themenfelder in integrativen Fächern der sozialwissenschaftlichen Domäne wie aktuell bei der Einführung des Faches ,Wirtschaft-Politik“ in NRW (Beyer 2019). Problematisch ist daran zumindest, dass sich Schülerinnen und Schüler auf diese Weise eben nicht mit drängenden Problemstellungen und Herausforderungen in Bezug auf den Gegenstandsbereich Wirtschaft auseinandersetzen, sondern eine spezifische Perspektive einüben, ohne sich dessen bewusst zu sein. Anders ausgedrückt handelt es sich um eine „Exklusion der Wirtschaft aus der ökonomischen Bildung“ (Graupe 2017b, S. 67). Lehramtsstudierende, die eine solche ökonomische Bildung von Schülerinnen und Schülern verantworten müssen, werden vielfach selbst nur mit dem monoparadigmatischen Zugriff der Wirtschaftswissenschaften konfrontiert, meist ohne Kenntnis und Reflexion der begrenzten erkenntnistheoretischen Reichweite ökonomischer Modellbildung. Zugespitzt formuliert drängt eine reduzierte neoklassische Ökonomik in die Schulbildung, ohne dass diejenigen, die eine solche Bildung verantworten müssen, selber darum wissen oder Bildungsräume für Alternativen erfahren können. An Universitäten, an denen Interdisziplinarität ernst genommen wird, erwerben Studierende weitere Perspektiven auf Wirtschaft etwa im Zuge soziologischer oder politikwissenschaftlicher Zugänge. Was jedoch in der Lehre viel-

\footnotetext{
${ }^{1}$ Hier ist als Beispiel das Fach „Wirtschaft. Berufs- und Studienorientierung“ zu nennen, welches in Baden-Württemberg zum Schuljahr 2017/2018 eingeführt wurde.
} 
fach fehlt, ist die dezidierte, kritische und reflektierte Auseinandersetzung mit der (neoklassisch geprägten) Ökonomik selbst.

\subsection{Das ,Wie': mangelnde Kontroversität und Etablierung von Wahrheiten}

Doch nicht nur das ,Was' ökonomischer Bildung stellt ein Problemfeld dar. Gerade in Grundfragen des Wirtschaftens vermögen Lehrmittel die einseitigen Inhalte überwiegend in Form von absolut gesetzten Wahrheiten statt in Form von Sinnhorizonten zu transportieren, welche die Denk- und Urteilskraft der Schülerinnen und Schüler fördern würden. Ganz wie in der VWL, wo die Lehrbuchautoren Samuelson und Nordhaus (2010, S. XVII) von „The core Truth of Economics", also von einer eindeutigen ökonomischen Wahrheit sprechen, besteht die Gefahr, eine Art und Weise auf die Welt zu blicken vorzugeben, die das Einüben unterschiedlicher Perspektiven systematisch zu unterbinden droht. Dieser Mangel an Kontroversität ist in der Schulbildung gemäß des Beutelsbacher Konsenses weder zulässig, noch trägt eine solche Bildung zum humanistischen Bildungsziel der Mündigkeit bei. Silja Graupe zeigt in einer 2017 veröffentlichen Studie auf, dass in der universitären Hochschulbildung sehr deutlich sprachliche Beeinflussungstechniken zum Tragen kommen, welche ehrliche Bildungserfahrungen unterminieren (Graupe 2017a). Eine solche Behinderung des Bildungszieles Mündigkeit findet sich nach eigenen Erhebungen ${ }^{2}$ auch in Schulbüchern und Open Educational Resources (OER). Dies gilt sowohl für aus akademischen Lehrbüchern in Schulbücher übernommene Auszüge als auch für direkt auf Bildungskontexte zugeschnittene Lehrmittel. Zudem tendieren Bildungsmaterialien im angesprochenen Themenbereich $\mathrm{zu}$ einer undurchsichtigen Vermengung von Modellen bzw. Theorien mit Welt, was zu fachlichen Mängeln führt. Ein aussagekräftiges Beispiel ist das Modell der Preisbildung: Eine erkenntnistheoretische Reflexion dessen, was dieses aussagen kann, weicht in vielen Fällen einer unreflektierten Anwendung der Preisbildung auf das Kaufen von Eiskugeln oder Hamburgern. Preisbildung wird so nicht als Modell besprochen und unterrichtet, welches bewusst realitätsferne, weil a priori gesetzte Annahmen einschließt, sondern vielmehr als hinzunehmender Fakt dargestellt, den es nicht zu hinterfragen gilt.

${ }^{2}$ Die Untersuchung wird im nächsten Jahr veröffentlicht. 
Tab. 1 Überblick über den Seminarverlauf. Quelle Eigene Darstellung

\begin{tabular}{l|l}
\hline 1 & Analyseraster in der Lehrmittelforschung \\
\hline 2 & $\begin{array}{l}\text { Die Bezugsdisziplin Wirtschaftswissenschaft: Gegenstandsbereich oder } \\
\text { Methode? }\end{array}$ \\
\hline 3 & Denken in Modellen und Realität - Wege zu einer transformativen Wissenschaft \\
\hline 5 & ,Der Markt' in Lehrbüchern, Lehrmitteln an Schulen und Diskursen \\
\hline 6 & $\begin{array}{l}\text { Kontroversität, Multiperspektivität \& Interdisziplinarität: Plurale Ökonomik und } \\
\text { sozioökonomische Bildung }\end{array}$ \\
\hline $7 . / 8$ & Fishbowl-Debatte: Das Fach Wirtschaft \\
\hline Block & $\begin{array}{l}\text { I. Lehrmittelwerkstatt: Sichtung, Analyse und Einordnung } \\
\text { II. Simulationen in sozioökonomischer Bildung: Erprobung, Chancen und } \\
\text { Grenzen }\end{array}$ \\
\hline 13 & Anders Wirtschaften? Alternative Ansätze im Vergleich \\
\hline 14 & Und jetzt...? Wie möchte ich Wirtschaft unterrichten? \\
\hline
\end{tabular}

\section{Entstehungskontext}

\subsection{Hintergrund und Gestaltung des Seminars}

Im Rahmen des Seminars „Ökonomie kritisch denken und unterrichten: Analyse, Sichtung und Erstellung von Lehrmitteln und Methoden der sozioökonomischen Bildung" habe ich versucht, den oben skizzierten Problemlagen ${ }^{3}$ des ,Was' und des ,Wie' in der ökonomischen Bildung konstruktiv zu begegnen und fachdidaktische Alternativen zu entwickeln. Dieses Seminar richtete sich an Bachelorstudierende mit dem Unterrichtsfach Sozialwissenschaften und fand an der Universität Bielefeld im Sommersemester 2019 statt. Maßgebliches Ziel des Seminars war es, plurale und erkenntnistheoretische Zugriffe auf die Bezugsdisziplin Ökonomik für fachliche Fragen und fachdidaktische Anliegen fruchtbar zu machen (einen Überblick zum Seminarverlauf bietet Tab. 1). Als fachdidaktisches Vertiefungsseminar fand diese Lehrveranstaltung unter der

\footnotetext{
${ }^{3}$ Ich konzentriere mich im Folgenden auf die Beschreibung derjenigen Seminaraspekte, die maßgeblich mit den beschriebenen Problemlagen zusammenhängen.
} 
besonderen Bedingung einer kleinen Lerngruppe von 15 Personen an einem der pluralen, sozialwissenschaftlichen Bildung sehr aufgeschlossenen Fachbereich der Universität Bielefeld statt. Als Grundlage brachten die Teilnehmenden den Besuch einer Einführungsveranstaltung in die VWL (10 ECTS) sowie einer Einführung in die Didaktik der Sozialwissenschaften (10 ECTS) mit. Es fand im wöchentlichen Turnus statt, wobei ein Blocktermin für aufwendigere Anwendungssituationen genutzt wurde. Ich habe das Seminar zum ersten Mal unterrichtet; eingeflossen sind Erfahrungen aus der Praxis als Lehrer sowie meine Auseinandersetzung mit fachdidaktischen und fachlichen Aspekten sozioökonomischer Bildung, die ich in einem Masterprogramm ${ }^{4}$ nach dem Referendariat vertieft habe.

\subsection{Grundlegende Aspekte der Seminargestaltung}

Im Rahmen des Seminars wurde mehrfach selbstverständlich Geglaubtes hinterfragt. Um dies in einem verantwortbaren Rahmen zu tun, ist eine offene Haltung gegenüber den Studierenden, ihren individuellen Fragen sowie ihren Möglichkeiten geboten. Als wichtige Voraussetzung sehe ich in diesem Zusammenhang an, den Fokus auf die Weiterentwicklung und Professionalisierung der Studierenden zu legen, die eine ernsthafte und intrinsisch motivierte Auseinandersetzung auch über das scheinbar Geforderte hinaus erlaubt. Hilfreich war es hier, Bewertungsräume sehr klar abzugrenzen und immer wieder flexibel auf die Bedürfnisse der Seminargruppe in Zeit- und Inhaltsgestaltung zu reagieren.

Für die Seminarsitzungen ergab sich daraus die Herausforderung, eine Atmosphäre des kooperativen Arbeitens und Denkens zu etablieren, und ein wirklich offenes Gespräch führen zu können, was als essentiell für das Gelingen des Seminars zurückgemeldet wurde. Dies habe ich in der ersten Sitzung mittels der sog. Vier-Ecken-Übung 5 eingeleitet und in den folgenden Sitzungen durch zwei banal erscheinende - aber, wie sich im Feedback der Studierenden gezeigt

\footnotetext{
${ }^{4}$ Es handelt sich um das Masterprogramm „Ökonomie und Gesellschaftsgestaltung“ an der Cusanus Hochschule für Gesellschaftsgestaltung in Bernkastel-Kues.

${ }^{5}$ Die Vier-Ecken-Übung: Hierbei wird den Teilnehmenden eine Frage mit vier Antwortmöglichkeiten gestellt. Zur Beantwortung und um ins Gespräch zu kommen ordnen sich die Teilnehmenden den jeweiligen Ecken zu. Ein Beispiel aus dem Seminar ist: „Wer oder was hat dein Denken über Wirtschaft am meisten beeinflusst? 1. Eltern, 2. Schule, 3. soziales Umfeld, 4. Literatur und Medien“.
} 
hat, grundlegende - Gelingensbedingungen für eine kooperative Haltung im gemeinsamen Arbeiten weiter zu ermöglichen gesucht: zum einen die Tischanordnung als gemeinsamer Gruppentisch und zum anderen explizite Metaphasen, in denen das gemeinsame Arbeiten reflektiert wurde. Zudem habe ich mich bemüht, genügend Gesprächsraum für individuelle Aspekte im Plenum, aber auch in Kleingruppen zur Verfügung zu stellen. In der Vor- und Nachbereitung zu den Seminarsitzungen haben die Studierenden Fragen und Aufgaben (über ein online-tool ${ }^{6}$ ) bearbeitet, in denen sie zur persönlichen Reflexion eingeladen wurden und vorbereitete Texte sowie kleine Aufgabenstellungen zur konstruktiven und entlastenden Zusammenarbeit während der Präsenzzeit bearbeiten konnten. Diese Aufgaben waren Teil der Erarbeitung eines rahmenden Portfolios, der Studienleistung des Seminars. Die erste Aufgabe, die es zum Seminarauftakt vorbereitend zu bearbeiten galt, war beispielsweise die persönliche Auseinandersetzung mit den Fragen ,Warum sollte Ökonomie an Schulen unterrichtet werden?“ sowie ,Warum möchtest Du Ökonomie an Schulen unterrichten?" in Form einer schriftlichen Kurzdiskussion. Interessant war hierbei, dass einige Studierende sich die Frage bisher kaum gestellt hatten und auf diese Weise motiviert wurden, herauszufinden, was sie da eigentlich wie unterrichten wollen beziehungsweise in vielen Fällen sollen. Für den inhaltlichen Aufbau des Seminars hat es sich als sehr fruchtbar erwiesen, fachdidaktische und fachwissenschaftliche Elemente konsequent zu verschränken: Haben wir uns mit fachlichen Aspekten beschäftigt, schloss beispielsweise eine thematisch passende Lehrmittelanalyse an; bei der Beschäftigung mit eher fachdidaktischen Fragen wurden fachliche Aspekte explizit in den Blick genommen.

\section{Innovative Lösung im Sinne einer pluralen, sozioökonomischen Hochschulbildung}

\subsection{Dem ,Was' begegnen: Ökonomik und Neoklassik reflektieren und einordnen}

„Warum sagt einem das niemand?“ fragte ein Student, nachdem wir uns über vier Sitzungen hinweg intensiv mit den Grundlagen derjenigen Art von standardökonomischem Denken beschäftigt haben, die Studierenden bereits im Studium

\footnotetext{
${ }^{6}$ Die zu erbringenden Aufgaben wurden am Ende des Seminars samt Reflexion als Portfolio zusammengefasst, welche die Studienleistung darstellt.
} 
vertieft kennengelernt haben. Doch wurden sie darüber im Dunkeln gelassen, was hinter den oben zitierten „,core truths“ zu liegen scheint. Der innovative Charakter der Vorgehensweise des Seminars liegt darin, das Scheinwerferlicht auf plurale Alternativen und epistemologische Fragen zu werfen.

Als Ausgangspunkt des Seminars diente uns ein Primärtext von Gary S. Becker, der in sehr pointierter Art und Weise den sogenannten „Economic Approach" (Becker 1990) formuliert hat, und der wie kein anderer für die Etablierung von Ökonomik als Methode, die Welt in allen Lebensbereichen zu betrachten, steht. Als Methode der Bearbeitung dieses dichten und deshalb intensiv vorzubereitenden Textes hat sich das close reading ${ }^{7}$ angeboten. Sich auf die von Becker (1990) vorgestellten Annahmen und seine Art und Weise, auf die Welt zu schauen, einzulassen, erforderte ein hohes Maß an Konzentration. Den Studierenden eröffneten sich grundlegende Einblicke, was es bedeutet, wenn Ökonomik neben der ,Wirtschaft' auch gesellschaftliche Funktionsbereiche wie Bildung, Liebe oder Pflegewesen zu erklären mag und dabei neoklassische Annahmen (etwa Nutzenmaximierung, atomistisches Menschenbild, Marktgleichgewicht) gewissermaßen zur Realität macht. Doch auch positive Aspekte dieser Art zu denken, etwa im Rahmen von Effizienzsteigerung, ohne diese als Selbstzweck zu begreifen, konnten unter der angeleiteten Einordnung von Annahmen sowie der Erkenntnisreichweite von Beckers Text gefunden werden. Im nächsten Schritt stand die erkenntnistheoretische Dimension im Mittelpunkt. Als sehr erkenntnisreich wurde vonseiten der Studierenden die kritische Reflexion des Denkens in Modellen und dessen Zusammenhang mit Welt empfunden. Wie eingangs angesprochen, ist diese Reflexion weder in Schulbüchern noch in den einschlägigen Einführungswerken der VWL zu finden, weshalb sie für die Studierenden vielfach ein neues Erkenntnismoment darstellte. Die Wechselwirkungen zwischen Welt und Modellen/Theorien sowie deren Erkenntnisgrenzen in Bezug auf die ökonomische Wirklichkeit wurden intensiv besprochen, visualisiert und beurteilt. Als hilfreich für das Verständnis hat sich neben einschlägiger Literatur (Barth und Rommel 2018; Brodbeck 2013; Hill und Myatt 2010) die Auseinandersetzung mit dem „Monetary National Income

\footnotetext{
${ }^{7}$ Die Methode des close reading (weitere Inforamtionen bei Hallet 2010) kommt ursprünglich aus der Literaturwissenschaft. Es geht um eine intensive und textgenaue Analyse und Interpretation eines Textes. Im vorliegenden Fall hat das Lesen und Interpretieren ausgewählter Stellen von sechs Seiten eine Stunde in Anspruch genommen.
} 
Analogue Computer"8 (MONIAC) - nach dem Erfinder Bill Phillips auch als „Phillips-Maschine“ bekannt - erwiesen. Sowohl die Vorführung des MONIAC in Form eines Videos als auch das Nachvollziehen des Modells anhand eines Fotos halfen, die grundlegende Idee der Modellhaftigkeit zu verdeutlichen. Beim Reden von ,dem Markt" kommt erschwerend hinzu, dass vielfach unklar bleibt, was mit diesem Begriff genau gemeint ist. Nicht nur im alltäglichen Sprachgebrauch mischen sich die Bilder und Assoziationen zu ,der Markt ${ }^{\star}$ - auch Schulbücher weisen häufig den fachlichen Mangel auf, Bedeutungen wie Wochenmarkt, Finanzmarkt oder vollkommener Markt zu vermischen, als Entität aufzufassen und dekontextualisiert als unpräzisen Containerbegriff $\mathrm{zu}$ verwenden (Ötsch 2019). Dies als Ausgangslage auffassend, konnte am Beispiel des Marktmodells mit Blick auf Annahmen eines vollkommenen Marktes und die in Schulbüchern gefundenen Anwendungsbeispiele reflektiert werden, wie ein konstruktiver Umgang mit Modellen im Verhältnis zur Realität in Bildungskontexten aussehen kann.

\subsection{Dem ,Was' begegnen: Alternativen auf verschiedenen Ebenen anbieten und aufzeigen}

Ursprünglich aufgrund der begrenzten Zeit nur als Ausblick vorgesehen, forderten die Studierenden eine deutliche Auseinandersetzung mit konstruktiven Alternativen ein. Denn die skizzierte Abhandlung ökonomischer Grundlagen ließ einige Studierende laut Selbstbeschreibung ,desillusioniert“ zurück. Ich kam der Bitte auf zwei Ebenen nach: Zum einen durch eine exemplarische Auseinandersetzung mit Multiparadigmatizität und Interdisziplinarität anhand des Mindestlohnes (1) (siehe Abb. 1) und zum anderen durch die Erarbeitung von Ansätzen alternativen Wirtschaftens (2).

Multiparadigmatizität und Interdisziplinarität als Grundpfeiler einer sozioökonomischen Bildung wurden auf die Frage angewendet, ob der gesetzliche Mindestlohn auf $12 €$ erhöht werden sollte. Befragt wurden verschiedene ökonomische Denkschulen (siehe Abb. 1) mit Blick auf grundlegende Argumentationslinien. Diskutiert wurde ebenfalls, inwiefern den Studierenden bekannte Zugriffe aus Soziologie und Politikwissenschaft eine Thematisierung

${ }^{8}$ Der Link zum Video des MONIAC findet sich hier unter, Online: https://www.youtube. com/watch?v=rVOhYROKeu4 [31.07.19]. 


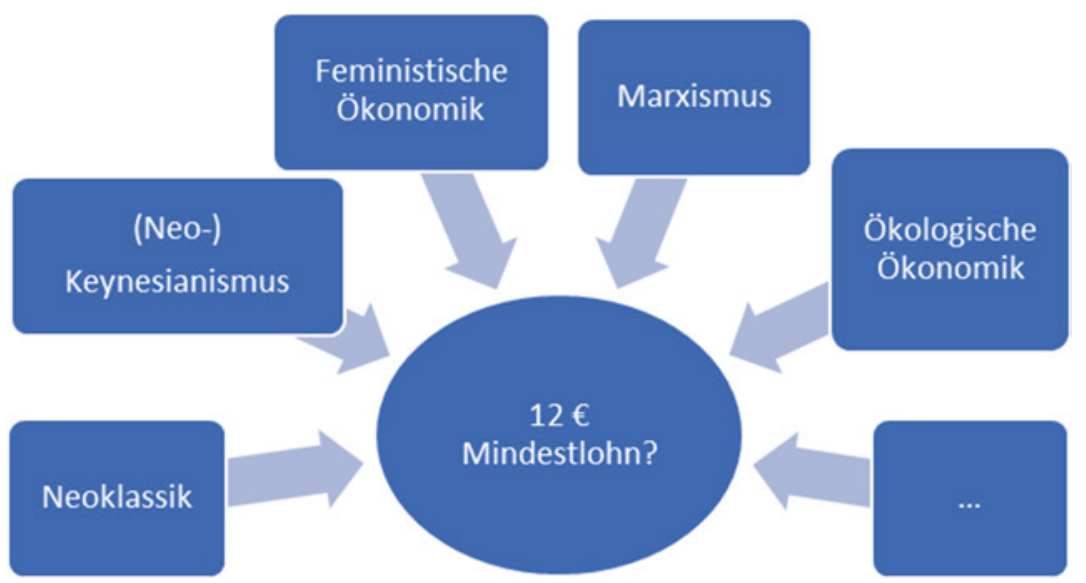

Abb. 1 Multiparadigmatizität am Beispiel Mindestlohn.

Quelle: eigene Darstellung

der Auswirkungen von Herrschafts- und Machtverhältnissen ermöglichen. Es zeigte sich klar, dass das konkrete problemorientierte multiperspektivische Arbeiten hilft, alternative Paradigmen besser kennen und anwenden zu lernen. Zur Entlastung der Studierenden sollte für die angemessene Einordnung der Theorieschulen deutlich mehr Zeit eingeplant werden, als für dieses Seminar veranschlagt wurde. Dies gilt auch aufgrund des Zeitbedarfs, den die Berücksichtigung interdisziplinärer Aspekte erfordert.

Auf einer ganz anderen Ebene beschäftigten sich die Studierenden arbeitsteilig mit „Alternativen des Wirtschaftens“. Nach einer Kurzvorstellung von meiner Seite wurden exemplarisch die Felder Degrowth, Commons, Gemeinwohl-Ökonomie (GWÖ), Grundeinkommen und Care Revolution ausgewählt. Als gemeinsame Grundlage diente die Textsammlung „Degrowth in Bewegungen“ (Konzeptwerk Neue Ökonomie und DFG-Kolleg Postwachstumsgesellschaften 2017), in der Alternativen des Wirtschaftens überblicksartig und mit vergleichbarem Umfang - für eine arbeitsteilige Vorbereitung durchaus relevant - vorgestellt werden. Die Konzepte wurden in Form eines Marktes der Möglichkeiten in den Austausch und ins Gespräch gebracht. Die Arbeitsgruppen zu den jeweiligen Themen erstellten Plakate mit der Maßgabe, über eine inhaltliche Zusammenfassung hinauszugehen. Schließlich folgte eine gemeinsame Vertiefung im Plenum zum Thema Gemeinwohl-Ökonomie (GWÖ). Mit der 
maßgeblich von Christian Felber entwickelten GWÖ-Matrix machten sich die Studierenden daran, einen geteilten Erfahrungsraum der Bewertung zu unterziehen, nämlich die Mensa der Universität. Kriteriengeleitet wurde so eine erste Einschätzung mit Bepunktung im Rahmen der GWÖ-Matrix vorgenommen. Hierbei wurden recht schnell blinde Flecken in Bezug auf ökologische Nachhaltigkeit und deutliche Stärken in punkto Solidarität der Bielefelder Mensa identifiziert. Durch das Erproben der Matrix und der anschließenden Diskussion der Erfahrungswerte im Umgang mit selbiger konnte auch klar reflektiert werden, wo Grenzen eines solchen Vorgehens liegen und wie realistisch eine auf gesamtwirtschaftliche Zusammenhänge gerichtete Skalierung der GWÖ einzuschätzen ist. Vorschnelle Einschätzungen wie „die Gemeinwohl-Ökonomie ist die Antwort auf all meine Fragen" wurden schließlich wieder zurückgenommen, da die Studierenden beispielsweise kritisch herausarbeiten konnten, dass weder die Wirtschaftsweise an sich noch das Denken über Ökonomie mit dem Konzept der GWÖ alleine fassbar oder gestaltbar sind.

\subsection{Dem ,Wie' begegnen: Pädagogische und Didaktische Doppeldecker}

Methodisch hilfreich war in vielerlei Hinsicht der Einsatz von sogenannten Pädagogischen und Didaktischen Doppeldeckern, die bereits angeklungen sind. Unter solchen „Doppeldeckern“ verstehe ich, „das, womit man sich inhaltlich beschäftigt, auch gleichzeitig zu erleben und wieder in die kognitive Auseinandersetzung mit dem Inhalt einzubeziehen“ (Geissler 1985, S. 8). Gerade in der Lehrerbildung bietet es sich an, für schulische Bildung vorgesehene Methoden und Unterrichtssettings im Rahmen von Seminaren zu erleben, zu erproben, studierendenseitig anzuleiten und einer kritischen Reflexion zu unterziehen. Diese Vorgehensweise prägte maßgeblich die Struktur der Seminarsitzungen. Auf die klassische Referatsform wurde verzichtet; Seminargestaltungen hatten den Anspruch motivierende und aktivierende Momente vorzuhalten. Um ein Beispiel zu geben: Zur Entwicklung der Urteilsbildung im schulischen Unterricht sind Debatten ein zentrales Strukturelement. Methodisch analog dazu wurde im Rahmen des Seminars von einer Gruppe Studierender eine Fishbowl-Debatte organisiert und umgesetzt, in der die aktuelle Frage „Sollte das Fach Wirtschaft an Schulen eingeführt werden?" fundiert und mitunter emotional aus vorgegebenen Rollen heraus diskutiert wurde. Die Textgrundlage samt Vertiefung habe ich hierbei bereitgestellt. Zudem ist es sehr ratsam, eine solche Stundengestaltung intensiv im Vorfeld zu besprechen. Zu meiner Überraschung 
war den Studierenden das Format zuvor fremd; sie meldeten überaus positiv zurück, dass die Sitzung ,wie im Flug vergangen“ sei und zuvor sicher geglaubte Meinungen deutlich auf den Prüfstand gestellt wurden.

Für die Rolle der Seminarleitung hat es sich durchaus bewährt, während der Umsetzung solcher Pädagogischer Doppeldecker strukturierend und nachhakend einzugreifen, auch wenn der Aktivitätsfluss hierdurch gebrochen wird. Hilfreich ist ein gedankliches ,freeze', um auf der Metaebene konzentriert und zügig nachzuhaken, wo Probleme und Herausforderungen liegen, also den ,Elefanten im Raum' zu benennen und zu vertreiben. In der Fishbowl-Debatte etwa wurden zunehmend eigene Vorstellungen eines Faches Wirtschaft mit monoparadigmatischen Konzeptionen vermischt; zudem wurde teilweise die Debattenrolle verlassen und die Debatte wurde auf die persönliche Ebene verschoben. Ein kurzzeitiges Ausbrechen aus der Situation durch eine moderierende Unterbrechung schaffte direkt Abhilfe.

Neben den eingesetzten Methoden bietet es sich auch an, Anforderungen für die schulische sozioökonomische Bildung im Sinne eines „Fachdidaktischen Doppeldeckers" nicht nur inhaltlich zu verhandeln, sondern konsequent für die Seminargestaltung ernst zu nehmen, soweit es möglich ist. Wie beschrieben, weicht so ein Reden über Kontroversität, Multiperspektivität oder Reflexivität einem Erleben und Einüben jener Prinzipien im Rahmen der inhaltlichen Auseinandersetzung.

\section{$4 \quad$ Folgen und Wirkung}

Die dem Seminar zugrunde liegende These, dass Historizität, Erkenntnisreichweite und Denkvoraussetzungen etablierter ökonomischer Standardmodelle erst kritisch zu durchschauen sind, bevor neues ökonomisches Denken entstehen kann, scheint sich zu bestätigen. Fragen nach einer lebensdienlichen Wirtschaft und zukunftsfähigen Ökonomien geraten so (wieder) in den Blick. Und es sind jene Fragen, welche im Seminar mit Blick auf Didaktik und Schulbildung angestoßen und bewegt wurden.

Auf Seite der Studierenden wurde ein hohes $\mathrm{Ma} \beta$ an Erkenntnisgewinn zurückgemeldet, das auch über die im Seminar behandelten Themen hinausging. So beschäftigen sich einige Studierende nun ,auch privat damit“ und sie fanden „ähnliche Problemlagen in Gesprächen mit Mitbewohnern“ auch in anderen Disziplinen und Feldern. 
Doch auch ein nicht zu unterschätzendes $\mathrm{Maß}$ an Verunsicherung auf Studierendenseite war im Seminarverlauf sehr herausfordernd und beschäftigte mich stark. Diese ist vor allem auf die Thematisierung von Theoriegrundlagen und erkenntnistheoretischen Aspekten sowie auf die Lehrmittelanalyse zurückzuführen. Diesbezüglich war es äußerst wichtig, Alternativen in Theorie und Praxis in das Seminar einzubauen und bei all den kritisierbaren Lehrmitteln und Inhalten auch konstruktive Ansätze zu vertiefen. Bei einer weiteren Durchführung des Seminars würde ich mich deutlicher bemühen, institutionelle Angebote für alternative ökonomische Ansätze (Vorlesungen, Netzwerke) aufzuzeigen. Außerdem würde ich in anderen Veranstaltungen entsprechende Vertiefungen einbauen.

Durchaus problematisch war der Eindruck von einigen Studierenden, vor allem kritisieren zu sollen, ohne dies selbst tiefer zu durchdenken. Auch wenn ich mir viel Mühe gegeben habe, für eine offene und selbstbestimmte Auseinandersetzung zu werben, so scheint ein Stolperstein zu sein, eine kritische Haltung als Anforderung misszuverstehen, dass vor allem Kritik zu üben sei. Zudem braucht es für die veranschlagten Themen und Fragen mehr Zeit als ein Seminar.

Es ist mir ein Anliegen, in der Lehrerbildung sowie der Schulbildung eine kritische und plurale Auseinandersetzung im Gegenstandsbereich Wirtschaft gemeinsam mit anderen weiter voranzubringen. Auf Grundlage meiner Erfahrungen und der anderer kann ich mir sehr gut vorstellen, als ersten Schritt einen Reader mit Umsetzungs- und Methodenvorschlägen sowie PPT-Folien für die universitäre Lehrerbildung zu erstellen. Zudem möchte ich auch institutionell mithelfen eine emanzipatorische, kritische und plurale sozioökonomische Bildung in Schule und Didaktik voranzubringen und noch mehr gelingende Beispiele einer guten sozioökonomischen Bildung in Schule und Hochschule zu finden und zu entwickeln. Ich freue mich also über Mitstreiterinnen und Mitstreiter, Ideen und konkrete Anfragen.

\section{Literatur}

Barth, J., \& Rommel, F. (2018). Auf dem Weg zu einer transformativen Wirtschaftswissenschaft. Kommunikations- und Institutionalisierungsstrategien für ein neues Wissenschaftsverständnis. In R. Pfriem, U. Schneidewind, J. Barth, S. Graupe \& T. Korbun, T. (Hrsg.), Transformative Wirtschaftswissenschaft im Kontext nachhaltiger Entwicklung (S. 561-589). Marburg: Metropolis.

Becker, G. (1990). The Economic Approach to Human Behavior. Chicago \& London: The University of Chicago Press.

Beyer, M. (2019). Erst Wirtschaft dann Politik? Neuer Name, neue Ausrichtung, bekannte Probleme. Stellungnahme zum Kernlehrplan (Entwurf Verbändebeteiligung: 
25.02.2019) des neuen Fachs „Wirtschaft-Politik“ in NRW. Gesellschaft für Bildung und Wissenschaft. https://bildung-wissen.eu/wp-content/uploads/2019/04/ Stellungnahme-KLP-G9-2019-Wirtschaft-Politik-GBW.pdf. Zugegriffen: 26. Juni 2019. Brodbeck, K.-H. (2013). Die fragwürdigen Grundlagen der Ökonomie (6. Aufl.). Darmstadt: WBG.

Engartner, T., Famulla, G., Fischer, A., Fridrich, C., Hantke, H., Hedtke, R., Weber, B., \& Zurstrassen, B. (2019). Was ist gute ökonomische Bildung? Leitfaden für den sozioökonomischen Unterricht. Frankfurt/M: Wochenschau.

Geissler, K. (1985). Lernen in Seminargruppen. Studienbrief 3 des Fernstudiums Erziehungswissenschaft „Pädagogisch-psychologische Grundlagen für das Lernen in Gruppen “. Tübingen: Deutsches Institut für Fernstudien.

Graupe, S. (2017a). Beeinflussung und Manipulation in der ökonomischen Bildung Hintergründe und Beispiele. Düsseldorf: Forschungsinstitut für gesellschaftliche Weiterentwicklung (FGW). FGW-Studie Neues Ökonomisches Denken 05. http://www. fgw-nrw.de/fileadmin/user_upload/NOED-Studie-05-Graupe-A1-komplett-Web.pdf. Zugegriffen: 23. Dez. 2019.

Graupe, S. (2017b). Bezugsdisziplin Wirtschaftswissenschaft? Politikum, 4, 67-68.

Hallet, W. (2010). Methoden kulturwissenschaftlicher Ansätze: Close Reading und Wide Reading. In V. Nünning, A. Nünning, \& I. Bauder-Begerow (Hrsg.), Methoden der literatur- und kulturwissenschaftlichen Textanalyse. Metzler: Stuttgart, J.B.

Hedtke, R. (2008). Ökonomische Denkweisen. Eine Einführung . Multiperspektivität, Alternativen, Grundlagen. Schwalbach/Ts: Wochenschau.

Hedtke, R. (2012). Die Wirtschaft in der Schule: Agendasetting, Akteure, Aktivitäten. Bielefeld: Universität Bielefeld. http://www.uni-bielefeld.de/soz/ag/hedtke/pdf/Hedtke_ Working-Paper_3.pdf. Zugegriffen: 26. Juli 2019.

Hill, R., \& Myatt, A. (2010). The Economics Anti-Textbook: A Critical Thinker's Guide to Microeconomics. Halifax: Fernwood Publishers [u.a.].

Konzeptwerk Neue Ökonomie \& DFG-Kolleg Postwachstumsgesellschaften (Hrsg.) (2017). Degrowth in Bewegung(en). 32 alternative Wege zur sozialökologischen Transformation. München: oekom.

Ötsch, W. (2019). Mythos Markt. Mythos Neoklassik. Das Elend des Marktfundamentalismus. Marburg: Metropolis.

Pies, I. (2017). Wider die Narreteien des Augenscheins - wie lange noch wollen wir die junge Generation mit elaborierter Halbbildung abspeisen? Diskussionspapier des Lehrstuhls für Wirtschaftsethik an der Martin-Luther-Universität Halle-Wittenberg, Nr. 2017-10. Halle: Lehrstuhl für Wirtschaftsethik an der Martin-Luther-Universität HalleWittenberg. http://wcms.itz.uni-halle.de/download.php?down=46319\&elem $=3066139$. Zugegriffen: 23. Dez. 2019.

Pühringer, S., \& Bäuerle, L. (2019). What Economics Education Is Missing: The Real World. International Journal of Social Economics, 46(8), 977-991.

Riedel, H. (2015). Politik \& Co., Politik und Wirtschaft für das Gymnasium. Neu. Hessen (1. Aufl.). Bamberg: CC. Buchner.

Samuelson, P., \& Nordhaus, W. (2010). Economics (19. Aufl.). New York, NY: McGraw-Hill. 
Open Access Dieses Kapitel wird unter der Creative Commons Namensnennung 4.0 International Lizenz (http://creativecommons.org/licenses/by/4.0/deed.de) veröffentlicht, welche die Nutzung, Vervielfältigung, Bearbeitung, Verbreitung und Wiedergabe in jeglichem Medium und Format erlaubt, sofern Sie den/die ursprünglichen Autor(en) und die Quelle ordnungsgemäß nennen, einen Link zur Creative Commons Lizenz beifügen und angeben, ob Änderungen vorgenommen wurden.

Die in diesem Kapitel enthaltenen Bilder und sonstiges Drittmaterial unterliegen ebenfalls der genannten Creative Commons Lizenz, sofern sich aus der Abbildungslegende nichts anderes ergibt. Sofern das betreffende Material nicht unter der genannten Creative Commons Lizenz steht und die betreffende Handlung nicht nach gesetzlichen Vorschriften erlaubt ist, ist für die oben aufgeführten Weiterverwendungen des Materials die Einwilligung des jeweiligen Rechteinhabers einzuholen.

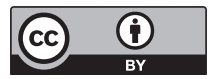

